\title{
Star clusters in the starburst galaxy M82
}

\author{
Sungsoon Lim, ${ }^{1}$ Narae Hwang ${ }^{1,2}$ and Myung Gyoon Lee ${ }^{1}$ \\ ${ }^{1}$ Department of Physics and Astronomy, Seoul National University, 599 Gwanak-ro, \\ Gwanak-gu, Seoul, Republic of Korea \\ email: [slim, mglee] @astro.snu.ac.kr \\ ${ }^{2}$ National Astronomical Observatory of Japan, 2-21-1 Osawa, Mitaka, Tokyo, Japan \\ email: narae.hwang@nao.ac.jp
}

\begin{abstract}
We present a study of star clusters in the starburst galaxy M82 using the $B V I$ mosaic images taken with the Hubble Space Telescope's Advanced Camera for Surveys. We have selected about 850 clusters with $V<23$ mag based on their morphological information. The brightest cluster is as bright as $V \sim 16.5 \mathrm{mag}\left(M_{V} \sim-11.2 \mathrm{mag}\right)$ and most clusters are fainter than $V=18 \mathrm{mag}\left(M_{V}=-9.7 \mathrm{mag}\right)$. The $V$-band luminosity function is represented by a power law with a slope $\alpha \sim-2.0$ in the range of $-9.5<M_{V}<-7$ mag. The star clusters in M82 are mainly distributed in the galaxy's disk. However, about a dozen clusters are found far from the disk and are considered to belong to the halo of M82. The color-magnitude diagrams of star clusters show that most star clusters are highly reddened. We have derived the age of the star clusters using a spectral-energy-distribution fitting method with Bruzual \& Charlot simple stellar population models. We discuss the age distribution as well as the photometric properties of the star clusters in regard to the formation history of the M82 cluster population.
\end{abstract}

Keywords. galaxies: individual (M 82), galaxies: star clusters

\section{Introduction}

M82 is the nearest starburst galaxy (except for IC10) and Hi observation revealed that it is interacting with M81 and NGC 3077 (Yun et al. 1994). There are numerous studies of the star clusters in this galaxy, but most covered only a small part of the galaxy (e.g., de Grijs et al. 2001; Melo et al. 2005, etc.). Recently, Mayya et al. (2008) presented a study of 653 star clusters in the disk and central region of M82, based on the Hubble Space Telescope (HST)/Advanced Camera for Surveys (ACS) data. They concluded that disruption of star clusters in M82 is affected by the mass and size of the clusters, using the mass estimates derived assuming that the mean ages of the nuclear and disk clusters are, respectively, 8 and 100 Myr. In this study, we present a study of the star clusters in a wide field covering the entire disk of M82.

\section{Data and method}

We used mosaic images obtained by the Hubble Heritage Team using HST's ACS Wide Field Channel (WFC) through the F435W $(B)$, F555W $(V)$ and F814W $(I)$ filters in March 2006 (Mutchler et al. 2007). The field of view is about $12^{\prime} \times 8^{\prime}$ centered on M82 and covers its entire optical disk.

We used SExtractor (Bertin \& Arnouts 1996) for star cluster detection and photometry. Since star clusters in M82 $(D \approx 3.5 \mathrm{Mpc}$; Lee et al. 2009) appear slightly extended in the HST images, we selected star cluster candidates automatically using the structural parameters given by SExTRACTOR (e.g., $2.5<$ FWHM $<40$ pixels, ellipticity $<0.8$, area $>40$ pixels). To select a clean cluster sample, however, we visually inspected 


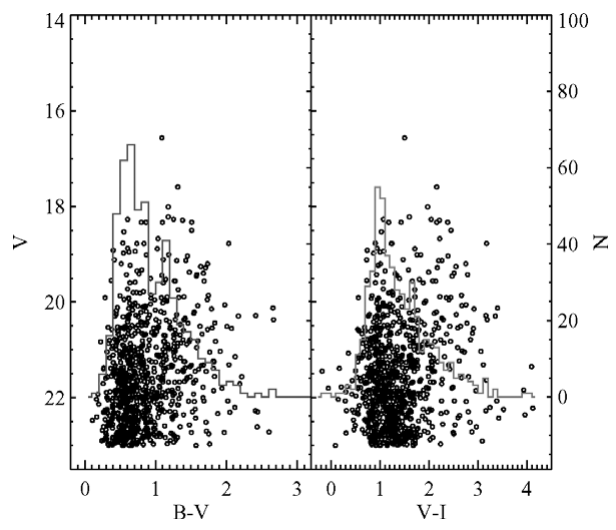

Figure 1. Color-magnitude diagrams (CMDs) of star clusters in M82. Solid lines show $(B-V)$ and $(V-I)$ color distributions for $V<22 \mathrm{mag}$, respectively.

the shapes of the radial profiles and 2D contours of star cluster candidates of $V<23$ mag. We compared the photometric data of clusters with the theoretical spectral-energy distribution (SED) to derive the age of the star clusters. SED models were produced using the population synthesis model of Bruzual \& Charlot (2003).

\section{Results}

We selected $\sim 850$ star clusters with $V<23$ mag based on visual classification. The brightest star cluster is as bright as $V \sim 16.5 \mathrm{mag}\left(M_{V} \sim-11.2 \mathrm{mag}\right)$. However, most are fainter than $V=18 \mathrm{mag}\left(M_{V}=-9.7 \mathrm{mag}\right)$. The color distribution of the star clusters shows two peaks at $(B-V) \sim 0.65$ and $(B-V) \sim 1.2 \mathrm{mag}$, respectively, and a long tail extending to the red (Figure 1$)$.

The color-magnitude diagram (CMD) of M82 star clusters displays somewhat different features from that of M51 star clusters (Hwang \& Lee 2008). The range of $(B-V)$ and $(V-I)$ colors of star clusters in M82 is much broader than that of star clusters in M51. It is suspected that this may be due to different extinctions and different inclinations between M82 and M51.

We have investigated the $V$-band luminosity function (LF) of the star clusters (Figure 2). The faint cut at $M_{V}=-4.7 \mathrm{mag}(V=23 \mathrm{mag})$ is the magnitude limit adopted for this study. The LF for $M_{V}>-7$ mag becomes flat, which is probably due to incompleteness effects. The logarithmic slope is $\sim-2.01 \pm 0.05$ in the range from -9.5 to $-6.5 \mathrm{mag}$, and is in a good agreement with the result of Mayya et al. (2008). It is found that the LF slope of M82 star clusters is similar to that of Small and Large Magellanic Cloud (SMC and LMC) star cluster populations. However, it is flatter than the LF slopes of star cluster systems in other spiral galaxies, suggesting that a larger number of very bright star clusters exist in M82 compared with other late-type galaxies.

Most of the star clusters are located in the galaxy disk, but some are found outside the disk (Figure 3). The red star clusters, with $(B-V)>0.9 \mathrm{mag}$, are mostly distributed in the central region of the disk, suggesting that they are highly reddened due to the internal extinction in the galaxy. There are also many bright star clusters in the central region of the disk. They are good candidates for super star clusters (SSC). However, some may be unresolved star clusters that are located close to one another. Since M82 is an edge-on galaxy, a distinction between disk and halo populations can be made rather easily. The red clusters in the halo are probably globular clusters. 


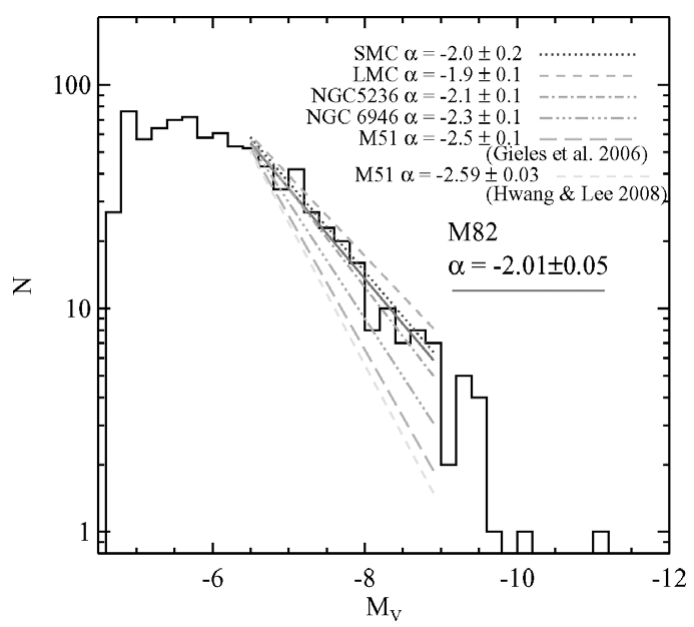

Figure 2. Luminosity function of star clusters in M82. The red solid line shows the power-law fit to the range $-9.5<M_{V}<-6.5 \mathrm{mag}$. The other lines show the luminosity function of star clusters in five other galaxies compiled from Gieles et al. (2006) and Hwang \& Lee (2008). Each luminosity function was shifted to match that of the M82 star clusters for easy comparison.

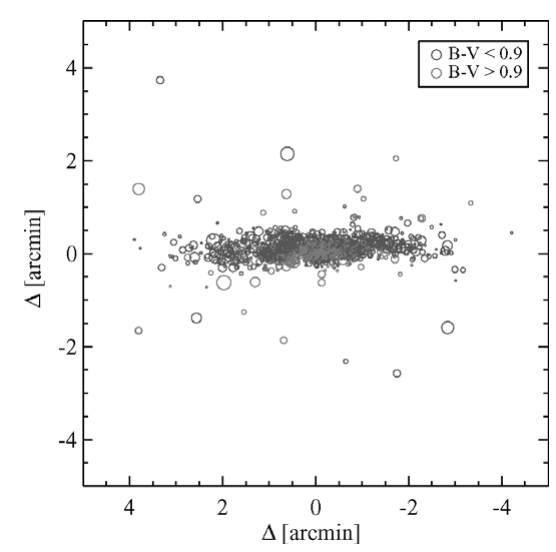

Figure 3. Spatial distribution of the star clusters. Larger symbols indicate brighter clusters. Blue and red open circles represent star clusters with $(B-V)<0.9$ and $>0.9$ mag, respectively.

The age distribution of star clusters shows three peaks, at $\log (t / \mathrm{yr})=6.5,8.8$ and $>10(3 \mathrm{Myr}, 630 \mathrm{Myr}$ and > $10 \mathrm{Gyr}$ ), respectively (Figure 4). The peak at $630 \mathrm{Myr}$ is consistent with the epoch of the last encounter with M81 expected from theoretical simulations (Yun 1999). The existence of young clusters with $\log (t / \mathrm{yr})=1(\sim 10 \mathrm{Myr})$ suggests that the recent star-formation episode has continued since about $10 \mathrm{Myr}$ ago. The oldest population, $\log (t / \mathrm{yr})>10$, may consist of globular clusters.

\section{Summary}

- We have found about 850 star clusters in M82. Most are located in the disk, but some $(\sim 3 \%)$ are found outside the disk.

- The logarithmic slope of the star cluster luminosity function is about -2.0 , similar to those for the SMC or LMC star cluster populations, but flatter than the luminosity function of M51 star clusters. 


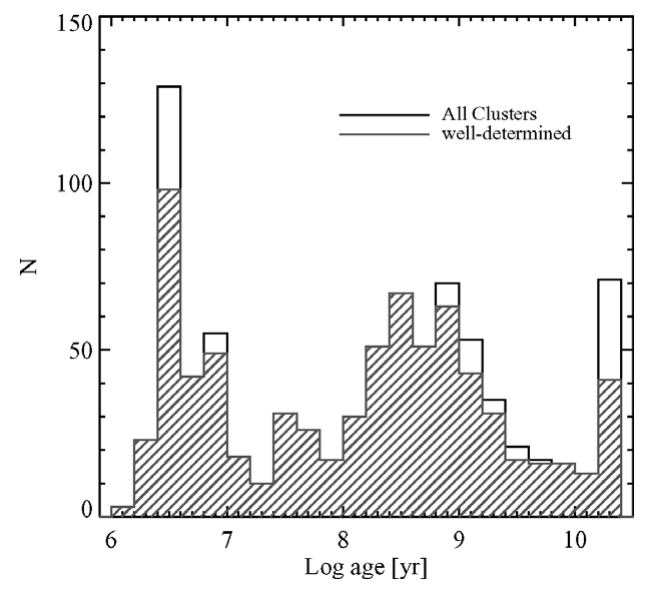

Figure 4. Age distribution of star clusters in M82. The blue shaded histogram represents star clusters with reliably fitted ages $\left(\right.$ small $\chi^{2}$ ). Note that there are three dominant populations of clusters, with $\log (t / \mathrm{yr})=6.5,8.8$ and $>10$.

- The peak for the intermediate-age population corresponds to the theoretically expected epoch of the last encounter with M81. It is also consistent with the result derived from star clusters in M81 by Chandar et al. (2001). This suggests that M81 and M82 may have undergone a strong interaction about 100 Myr ago, triggering the formation of clusters in both galaxies.

\section{Acknowledgements}

This work is supported in part by a grant (R01-2007-000-20336-0) from the Basic Research Program of the Korea Science and Engineering Foundation. NH acknowledges support by a Grant-in-Aid for JSPS Fellows, No. 20-08325.

\section{References}

Bertin, E. \& Arnouts, S. 1996, A\&SAS, 117, 393

Bruzual, G. \& Charlot, S. 2003, MNRAS, 344, 1000

Chandar, R., Tsvetanov, Z., \& Ford, H. C. 2001, AJ, 122, 1342

de Grijs, R., O'Connell, R. W., \& Gallagher III, J. S. 2001, AJ, 121, 768

Gieles, M., Larsen, S. S., Bastian, N., \& Stein, I. T. 2006, A\&A, 450, 129

Hwang, N. \& Lee, M. G. 2008, AJ, 135, 1567

Lee, M. G., et al. 2009, in prep.

Lim, S. 2008, MSc Thesis, Seoul National University

Mayya, D., Romano, R., Rodríguez-Merino, L. H., Luna, A., Carrasco, L., \& Rosa-González, D. 2008, ApJ, 679, 404

Melo, V. P., Muñoz-Tuñón, C., Maíz-Apellániz, J., \& Tenorio-Tagle, G. 2005, ApJ, 619, 270

Mutchler, M., et al. 2007, PASP, 119, 1

Yun, M. S., Ho, P. T. P., \& Lo, K. Y. 1994, Nature, 372, 530

Yun, M. 1999, in: J. E. Barnes \& D. B. Sanders (eds.), Galaxy Interactions at Low and High Redshift, Proc. IAU Symp. No. 186, p. 81 